\title{
Improvement of Sol-gel Method and Influence of Calcination Conditions on Properties of $\mathrm{MnO}_{\mathrm{x}}-\mathrm{CeO}_{\mathrm{x}} / \mathrm{WO}_{3} / \mathrm{TiO}_{2}-\mathrm{ZrO}_{2}$ Catalyst
}

\author{
Huixian Pan ${ }^{1}$, Li Wang ${ }^{1,2}$, , Shuanling He ${ }^{1}$, Jie Wang ${ }^{1}$ \\ ${ }^{1}$ Energy and Environmental Engineering School, University of Science and Technology Beijing, Beijing, China \\ ${ }^{2}$ Key Laboratory of Resource-Oriented Treatment of Industrial Pollutants, University of Science and Technology Beijing, Beijing, China \\ Email address: \\ 973234219@qq.com (Huixian Pan),wangli@ces.ustb.edu.cn (Li Wang), 862504844@qq.com (Shuanling He), \\ 1365206788@qq.com (Jie Wang) \\ ${ }^{*}$ Corresponding author
}

\section{To cite this article:}

Huixian Pan, Li Wang, Shuanling He, Jie Wang. Improvement of Sol-gel Method and Influence of Calcination Conditions on Properties of $\mathrm{MnO}_{\mathrm{x}}-\mathrm{CeO}_{\mathrm{x}} / \mathrm{WO}_{3} / \mathrm{TiO}_{2}-\mathrm{ZrO}_{2}$ Catalyst. Science Discovery. Vol. 5, No. 6, 2017, pp. 463-468. doi: 10.11648/j.sd.20170506.22

Received: October 2, 2017; Accepted: November 2, 2017; Published: November 21, 2017

\begin{abstract}
Modified sol-gel method was adopted to prepare $\mathrm{TiO}_{2}-\mathrm{ZrO}_{2}$ carrier of molar ratio 4:1, and the catalysts were prepared by impregnating with $\mathrm{WO}_{3}$ and $\mathrm{MnO}_{\mathrm{x}}-\mathrm{CeO}_{\mathrm{x}}$ sequentially. The carriers and catalysts were characterized by $\mathrm{X}$ ray diffraction (XRD) and electron scanning electron microscopy (SEM), and their $\mathrm{NH}_{3}-\mathrm{SCR}$ denitrification activity and sulfur resistance were investigated at low temperature. The results shown that mixing zirconium oxychloride with butyl titanate ethanol solution first and then titrating acetic acid inhibitor would have more $\mathrm{Zr}^{4+}$ substituted $\mathrm{Ti}^{4+}$ doping into the $\mathrm{TiO}_{2}$ lattice, resulting in more lattice defects and active sites. The surface morphology of carriers were all nano particles, but the carrier prepared by modified method shown smaller particle size and better dispersion. The catalyst which was calcined at $200^{\circ} \mathrm{C}$ for $2.5 \mathrm{~h}$ exhibited best $\mathrm{NH}_{3}$-SCR performance, its denitrification activity reached $98 \%$ at $100^{\circ} \mathrm{C}$, and the $\mathrm{NO}_{\mathrm{x}}$ conversion could be maintained at about $75 \%$ after continuously injecting $\mathrm{SO}_{2}(100 \mathrm{ppm})$ for $8 \mathrm{~h}$.
\end{abstract}

Keywords: Sol-Gel, Selective Catalytic Reduction, $\mathrm{TiO}_{2}, \mathrm{ZrO}_{2}$

\section{溶胶凝胶法的改进及煅烧条件对 $\mathrm{MnO}_{\mathrm{x}}-\mathrm{CeO}_{\mathrm{x}} / \mathrm{WO}_{3} / \mathrm{TiO}_{2}-\mathrm{ZrO}_{2}$ 催 化剂性能的影响}

潘惠贤 ${ }^{1}$, 汪莉 ${ }^{1,2}{ }^{*}$ ，贺拴玲 ${ }^{1}$, 王杰 ${ }^{1}$

1能源与环境工程学院, 北京科技大学, 北京, 中国

${ }^{2}$ 北京市工业典型污染物资源化处理重点实验室，北京科技大学，北京，中国

邮箱

973234219@qq.com（潘惠贤）, wangli@ces.ustb.edu.cn（汪莉）, 862504844@qq.com（贺拴玲），1365206788@qq.com（王杰）

摘要: 采用改良的溶胶凝胶法制备摩尔比 $4: 1$ 的 $\mathrm{TiO}_{2}-\mathrm{ZrO}_{2}$ 载体, 以典型的分步浸渍法依次浸渍负载 $\mathrm{WO}_{3} 、 \mathrm{MnO}_{\mathrm{x}}-\mathrm{CeO}_{\mathrm{x}}$, 制备催化剂。通过X射线衍射（XRD），电子扫描电镜（SEM）等技术手段对载体和催化剂进行表征，并考察其低温 $\mathrm{NH}_{3}$-SCR脱硝活性和抗硫性。结果表明, 先将氧氯化锆与钛酸丁酯乙醇溶液混合再滴定乙酸抑制剂, 会有更多的 $\mathrm{Zr}^{4+}$ 掺杂进入 $\mathrm{TiO}_{2}$ 晶格内, 产生较多的晶格缺陷和活性位点; 制备的载体均为纳米级颗粒, 改进制备方法后的载体粒径更

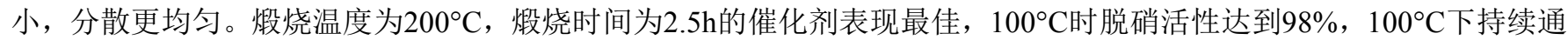
入 $\mathrm{SO}_{2}(100 \mathrm{ppm}) 8 \mathrm{~h}$ 后, $\mathrm{NO}$ 转化率可维持在 $75 \%$ 左右。 
关键词: 溶胶凝胶法, 选择性催化还原, $\mathrm{TiO}_{2}, \mathrm{ZrO}_{2}$

\section{1. 引言}

氮氧化物 $\left(\mathrm{NO}_{\mathrm{x}}\right)$ 包括多种化合物, 如 $\mathrm{NO} 、 \mathrm{NO}_{2} 、 \mathrm{~N}_{2} \mathrm{O}$ 等, $\mathrm{NO}_{\mathrm{x}}$ 能引起酸雨、光化学烟雾及 $\mathrm{PM} 2.5$, 是大气中主 要污染物之一。 $\mathrm{NO}_{\mathrm{x}}$ 的来源主要有移动源排放和固定点源 排放, 选择性催化还原 ( $\mathrm{SCR}$ ) 技术是控制固定源 $\mathrm{NO}_{\mathrm{x}}$ 排 放的主要技术, 其典型的商用催化剂是 $\mathrm{V}_{2} \mathrm{O}_{5}--\mathrm{WO}_{3}\left(\mathrm{MO}_{3}\right)$ $/ \mathrm{TiO}_{2}$ [1], 但是其活性窗口较高, 在 $300 \sim 400^{\circ} \mathrm{C}$ [2], 为此 需将 $\mathrm{SCR}$ 反应器置于除尘、脱硫之前, 烟气中高浓度的 $\mathrm{SO}_{2}$ 和粉尘极易造成催化剂的磨损和中毒[3]。经过除尘、半干 法脱硫之后的烟气温度约为 $80 \sim 150^{\circ} \mathrm{C}[4]$, 研究低温催化 活性较高且经济型较好的SCR催化剂具有重要意义。

$\mathrm{Mn}$ 系催化剂因为具有较高的 $\mathrm{NH}_{3}-\mathrm{SCR}$ 低温反应活性 [5], 成为研究热点, 主要分为非载体型和载体型催化剂两 大类。 $\mathrm{TiO}_{2}$ 负载的 $\mathrm{MnO}_{\mathrm{x}}$ 催化剂在低温 $\mathrm{NH}_{3}$-SCR脱硝反应 中表现出良好的催化性能, 应用最广。 $\mathrm{TiO}_{2}$ 具有较大的比 表面积, 利于活性组分的分散, 利于SCR反应的进行 [6]。 而 $\mathrm{ZrO}_{2}$ 同时拥有酸性、碱性、氧化性和还原性, 作为助催 化剂和晶型转化抑制剂得到广泛的应用。以共沉淀法制备 的 $\mathrm{TiO}_{2}-\mathrm{ZrO}_{2}$ 复合载体较之单纯的氧化物比表面积明显提 高, 可达 $218 \mathrm{~m}^{2} \cdot \mathrm{g}^{-1}$ [7]。 $\mathrm{TiO}_{2}-\mathrm{ZrO}_{2}$ 载体保持了 $\mathrm{TiO}_{2}$ 原有较 强的抗硫性能, 而且热稳定性和比表面积有所提高[8]。另 有研究[9]表明, $\mathrm{ZrO}_{2}$ 掺杂可以增强 $\mathrm{TiO}_{2}$ 的Lewis酸性。 $\mathrm{WO}_{3}$ 能够拓展催化剂的表面积, 增加催化剂表面的酸性位, 对 $\mathrm{MnO}_{\mathrm{x}}-\mathrm{TiO}_{2}$ 催化剂有很好的改性作用 $[10]$ 。 $\mathrm{Ce}$ 元素以 $\mathrm{Ce}^{4+}$ 、 $\mathrm{Ce}^{3+}$ 存在于 $\mathrm{Ce}-\mathrm{MnO}_{\mathrm{x}} / \mathrm{TiO}_{2}-\mathrm{ZrO}_{2}$ 催化剂中, 在反应中能起到 传递电子、离子和储氧的作用, 提高催化剂的氧化还原能 力 $[11]$ 。

本文在课题组研究的基础上, 采用溶胶凝胶法制备摩 尔比为 $4: 1$ 的 $\mathrm{TiO}_{2}-\mathrm{ZrO}_{2}$ 载体, 并对制备方法进行改进以优 化其性能, 通过XRD, SEM手段对载体进行表征, 并结合 其在 80 $200^{\circ} \mathrm{C}$ 下的活性测试结果, 得到性能最佳的 $\mathrm{TiO}_{2}-\mathrm{ZrO}_{2}$ 载体。最后对 $\mathrm{MnO}_{\mathrm{x}}-\mathrm{CeO}_{\mathrm{x}} / \mathrm{WO}_{3} / \mathrm{TiO}_{2}-\mathrm{ZrO}_{2}$ 催化剂 的㷽烧条件进行了篮选。

\section{2. 实验}

\section{1. 载体和催化剂的制备}

\subsection{1. 载体的制备}

采用溶胶凝胶法制备 $\mathrm{TiO}_{2}-\mathrm{ZrO}_{2}$ 载体, 共以三种方式 制备以优化其性能。
原方法: 将适量钛酸丁酯与无水乙醇混合形成A液, 一定量氧氯化锆与无水乙醇混合形成B液, 将冰醋酸、蒸 馏水和无水乙醇按一定体积比混合形成C液, 搅拌均匀。 搅拌中用滴定管将 $\mathrm{C}$ 液滴入 $\mathrm{A}$ 液, 再将 $\mathrm{B}$ 液缓慢加入。继续 搅拌, 得到均匀的淡黄色溶胶, 在室温下老化 $72 \mathrm{~h}$, 烘箱 中 $105^{\circ} \mathrm{C}$ 干燥 $24 \mathrm{~h}$ 得黄色晶体, 研磨后放入马弗炉中 $500^{\circ} \mathrm{C}$ 煅烧 $3 \mathrm{~h}$, 最终得到 $\mathrm{TiO}_{2}-\mathrm{ZrO}_{2}$ 载体, 记为 $\mathrm{TiO}_{2}-\mathrm{ZrO}_{2}(\mathrm{Y})$ 。

改进滴定法: 将 $\mathrm{A}$ 液和 $\mathrm{B}$ 液混合摚拌, 再将 $\mathrm{C}$ 液滴入混 合, 其它内容与原方法相同。得到载体记为 $\mathrm{TiO}_{2}-\mathrm{ZrO}_{2}(\mathrm{D})$ 。 改进反滴定: 将A液和B液混合摚拌, 滴入C液混合, 其它内容与原方法相同。得到载体记为 $\mathrm{TiO}_{2}-\mathrm{ZrO}_{2}(\mathrm{~F})$ 。

\subsection{2. 催化剂的制备}

采用典型的分步浸渍法制备 $\mathrm{MnO}_{\mathrm{x}}(\mathrm{x})-\mathrm{CeO}_{\mathrm{x}}(\mathrm{n}) / \mathrm{WO}_{3} / \mathrm{TiO}_{2}-\mathrm{ZrO}_{2}$ 催化剂。负载W: 将一定 量偏铇酸铵 ( $\left.\left(\mathrm{NH}_{4}\right)_{6} \mathrm{H}_{2} \mathrm{~W}_{12} \mathrm{O}_{40}\right)$ 溶于一定浓度的柠檬酸 溶液中, 在室温下匀速搅拌, $20 \mathrm{~min}$ 后加入适量载体 (使 $\mathrm{W}$ 元素负载量为 $10 \%$ ）, 继续摚拌, 超声, $70^{\circ} \mathrm{C}$ 水浴摚拌 $60 \mathrm{~min}$, 继而置于烘箱中 $90^{\circ} \mathrm{C}$ 干燥 $12 \mathrm{~h}$, 研磨后放入马弗炉 中, 在 $500^{\circ} \mathrm{C}$ 下㷽烧 $3 \mathrm{~h}$, 得到 $\mathrm{WO}_{3} / \mathrm{TiO}_{2}-\mathrm{ZrO}_{2}$ 催化剂。负载 $\mathrm{Mn}-\mathrm{Ce}$ : 将适量硝酸锰 $\left(\mathrm{Mn}\left(\mathrm{NO}_{3}\right)_{2}\right)$ 和硝酸铈 $\left(\mathrm{Ce}\left(\mathrm{NO}_{3}\right) \cdot 6 \mathrm{H}_{2} \mathrm{O}\right)$ 溶于一定浓度的柠檬酸溶液中, 浸渍步 骤与负载 $\mathrm{W}$ 相同 (使 $\mathrm{Mn}$ 元素负载量为 $5 \%, \mathrm{Ce} 、 \mathrm{Mn}$ 摩尔比 为 0.2 ), 搬烧温度和时间有所改变。得到 $\mathrm{MnO}_{\mathrm{x}}-\mathrm{CeO}_{\mathrm{x}} / \mathrm{WO}_{3} / \mathrm{TiO}_{2}-\mathrm{ZrO}_{2}$ 催化剂。

\section{2. 催化剂的表征}

(1) X射线衍射分析（XRD）: 采用Rigaku（日本理 学) DMAX-RB12kW旋转阳极X射线衍射仪分析催化剂颗 粒的物相。在 $40 \mathrm{~mA}$ 的电流强度、 $40 \mathrm{kV}$ 加速电压下, 采用 铜靶（波长为 $0.15406 \mathrm{~nm}$ ）, 并以 $20 \% / \mathrm{min}$ 的速度扫描, 其 扫描角度是 $2 \theta=10^{\circ} \sim 100^{\circ}$ 。

（2）催化剂电镜扫描分析（SEM）：采用美国FEI 公司的Quanta 3D FEG, 在 $10 \mathrm{kV}$ 的条件下, 进行电镜扫描, 观察催化剂的表面形貌。

\section{3. 催化剂活性测试}

催化剂性能测试在固定床反应器中进行, 反应装置如 图1所示。反应器为内径 $7 \mathrm{~mm}$ 的石英管, 催化剂所处为恒 温区, 由预热箱式电阻炉加热, 称取 $0.5 \mathrm{~g}$ 样品进行测试, 模拟烟气组成为: $0.067 \% \mathrm{NO}, \mathrm{Vol}\left(\mathrm{NH}_{3}: \mathrm{NO}\right)=1.1,6 \% \mathrm{O}_{2}$, $100 \mathrm{ppmSO}_{2}, \mathrm{~N}_{2}$ 为平衡气, 气体流量 $300 \mathrm{~mL} / \mathrm{min}$, 空速 $36000 \mathrm{~h}^{-1}$ 。 


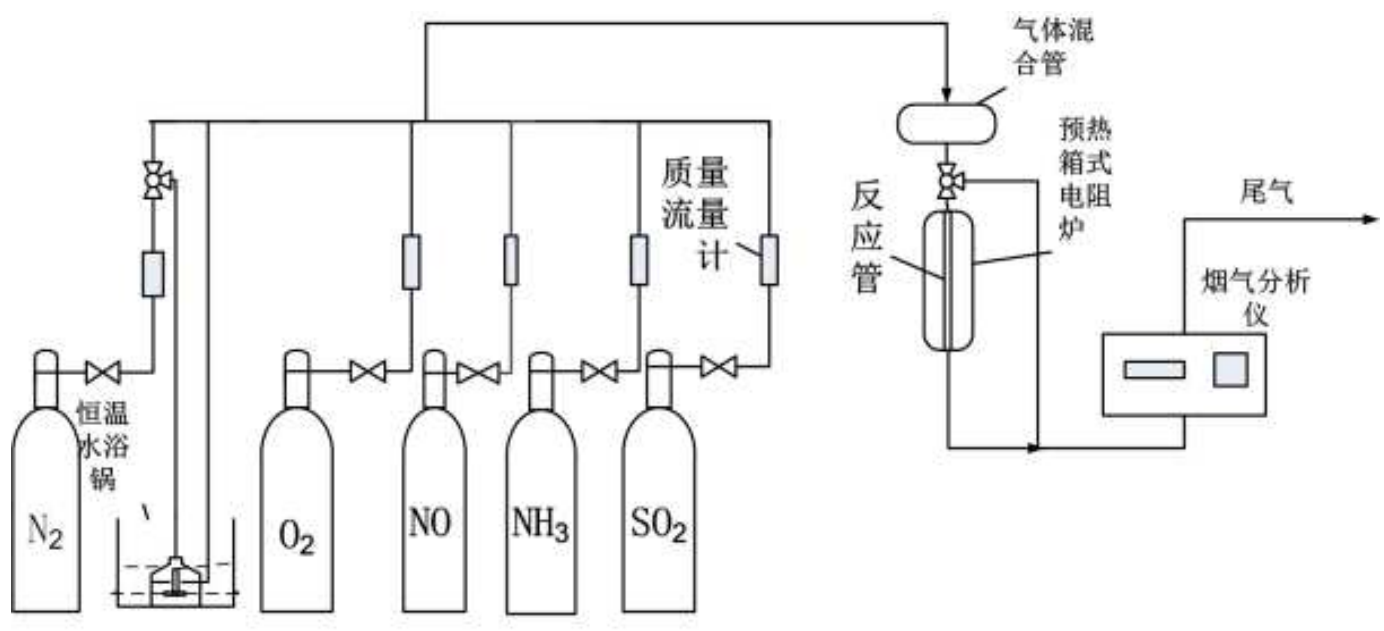

图1 SCR反应实验装置图。

$\mathrm{NO}_{\mathrm{x}}$ 的进出口浓度由烟气分析仪 (thermo scientific model 42i-HL) 测得, 用公式(1)计算催化剂的 $\mathrm{NO}_{\mathrm{x}}$ conversion:

$$
N O_{x} \operatorname{conversion}(\%)=\frac{\left[N O_{x}\right]_{(i n)}-\left[N O_{x}\right]_{(o u t)}}{\left[N O_{x}\right]_{(i n)}} \times 100 \%
$$

\section{3. 结果与讨论}

\section{1. 载体和催化剂的表征}

\subsubsection{XRD表征}

图2为 $\mathrm{TiO}_{2}-\mathrm{ZrO}_{2}(\mathrm{Y}), \mathrm{TiO}_{2}-\mathrm{ZrO}_{2}(\mathrm{~F})$ 和 $\mathrm{TiO}_{2}-\mathrm{ZrO}_{2}(\mathrm{D})$ 的 $\mathrm{X}$ 射线衍射图谱。从以下两个方面进行分析:

一方面, 三种制备方法所得 $\mathrm{TiO}_{2}-\mathrm{ZrO}_{2}$ 的XRD谱图中, 所有衍射峰均归属于锐铁矿 $\mathrm{TiO}_{2}$, 说明 $\mathrm{Zr}^{4+}$ 的添加没有导 致第二相产生。但相对于标准锐钛矿 (JCPDS 65-5714), 峰位均向小角度发生偏移, 比如, 标准锐铁矿对应于 (101) 晶面的角度为 $2 \theta=25.3^{\circ}$, 而 $\mathrm{a} 、 \mathrm{~b} 、 \mathrm{c}$ 谱图中对应的角度分别 为 $2 \theta=25.060^{\circ}, 25.060^{\circ}, 24.899^{\circ}$, 这可能是由于 $\mathrm{Zr}^{4+}$ (有 效半径 $0.072 \mathrm{~nm}$ ) 取代 $\mathrm{Ti}^{4+}$ (有效半径 $0.0605 \mathrm{~nm}$ ) 掺杂进入 $\mathrm{TiO}_{2}$ 晶格内, 导致 $\mathrm{TiO}_{2}$ 晶格常数变大, 引起晶格畸变, 产 生了更多的晶格缺陷和活性位点, $\mathrm{TiO}_{2}-\mathrm{ZrO}_{2}(\mathrm{D})$ 载体角度 偏移量最大。

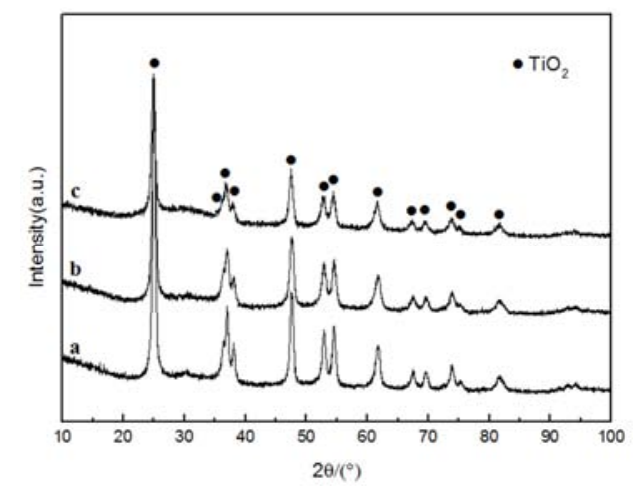

(a). $\mathrm{TiO}_{2}-\mathrm{ZrO}_{2}(\mathrm{Y})(\mathrm{b}) \cdot \mathrm{TiO}_{2}-\mathrm{ZrO}_{2}(\mathrm{~F})(\mathrm{c}) \cdot \mathrm{TiO}_{2}-\mathrm{ZrO}_{2}(\mathrm{D})$ 图2 不同载体的XRD谱图。
另一方面, 三种制备方法所得载体的峰强由大到小顺 序为 $\mathrm{TiO}_{2}-\mathrm{ZrO}_{2}(\mathrm{Y})>\mathrm{TiO}_{2}-\mathrm{ZrO}_{2}(\mathrm{~F})>\mathrm{TiO}_{2}-\mathrm{ZrO}_{2}(\mathrm{D})$, 利用 Scherrer公式对 $\mathrm{TiO}_{2}$ 衍射峰占比最大的 (101) 晶面进行计 算可得, $\mathrm{TiO}_{2}-\mathrm{ZrO}_{2}(\mathrm{Y}), \mathrm{TiO}_{2}-\mathrm{ZrO}_{2}(\mathrm{~F})$ 和 $\mathrm{TiO}_{2}-\mathrm{ZrO}_{2}(\mathrm{D})$ 的平 均粒径分别为 $15.94 \mathrm{~nm}, 13.21 \mathrm{~nm}, 11.89 \mathrm{~nm}$, 说明 $\mathrm{TiO}_{2}-\mathrm{ZrO}_{2}(\mathrm{D})$ 晶粒受抑制程度最大, 粒径最小。

\section{1.2. SEM表征}

图3为三种不同方法制备的 $\mathrm{TiO}_{2}-\mathrm{ZrO}_{2}$ 的扫描电镜图。 由图可见, 三种方法制备的载体晶粒均为纳米级, 在 10 20nm之间, 但相对来说, $\mathrm{TiO}_{2}-\mathrm{ZrO}_{2}(\mathrm{D})$ 载体晶粒更加 细小, 而且分布均匀。与XRD表征结果相符。

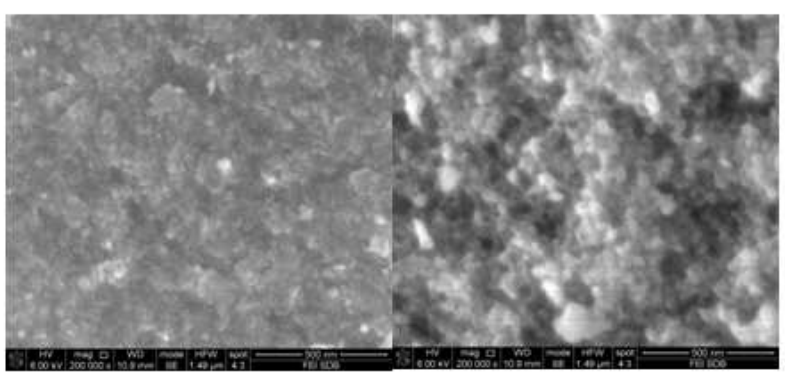

(a). $\mathrm{TiO}_{2}-\mathrm{ZrO}_{2}(\mathrm{Y})$

(b) $\mathrm{TiO}_{2}-\mathrm{ZrO}_{2}(\mathrm{~F})$

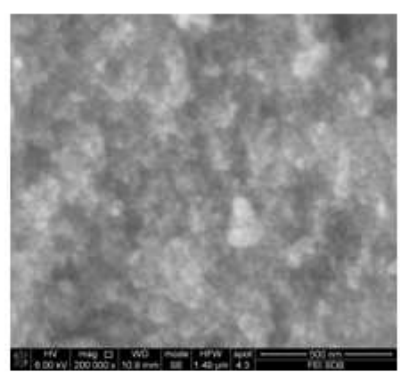

(c) $\mathrm{TiO}_{2}-\mathrm{ZrO}_{2}$ (D)

图3 不同载体的SEM电子扫描电镜图。 


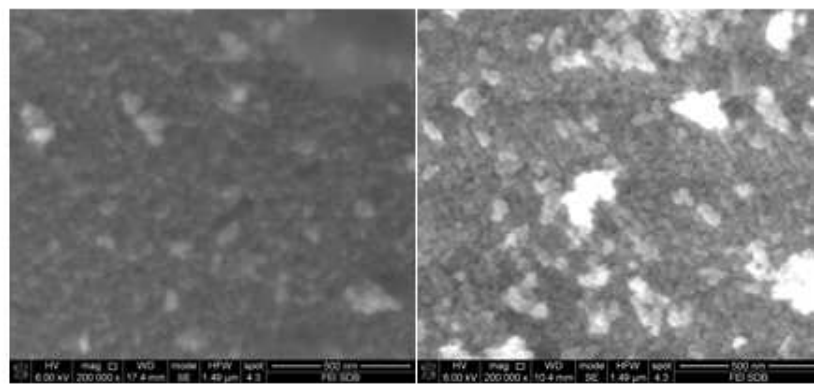

a. $150^{\circ} \mathrm{C}$

b. $200^{\circ} \mathrm{C}$

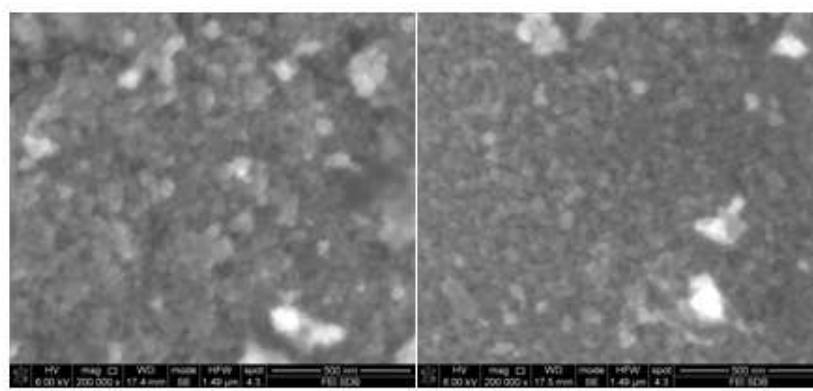

c. $250^{\circ} \mathrm{C}$

d. $300^{\circ} \mathrm{C}$

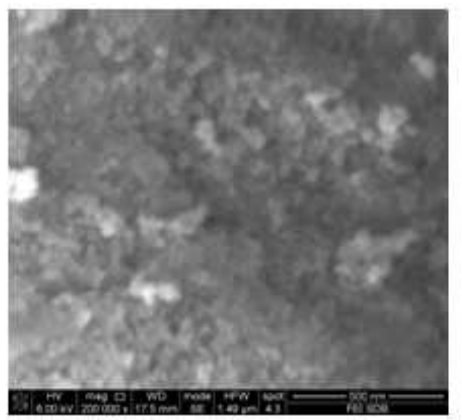

d. $350^{\circ} \mathrm{C}$

图4 不同煅烧温度催化剂的SEM图。

图5所示为不同煅烧时间催化剂的SEM电子扫描电镜 图。a、b、c、 $d 、 e$ 分别为煅烧时间为 $2 h 、 2.5 h 、 3 h 、 3.5 h$ 、 $4 \mathrm{~h}$ 的催化剂。从图中可以看出, 不同炦烧温度的催化剂表 面形貌均为纳米级颗粒, 从图中可以看出, 煅烧时间为 $2.5 \mathrm{~h}$ 的催化剂样品颗粒尺寸最小, 分散最均匀, 有利于 SCR 反应的进行, 其它催化剂颗粒出现了明显的团聚现象。

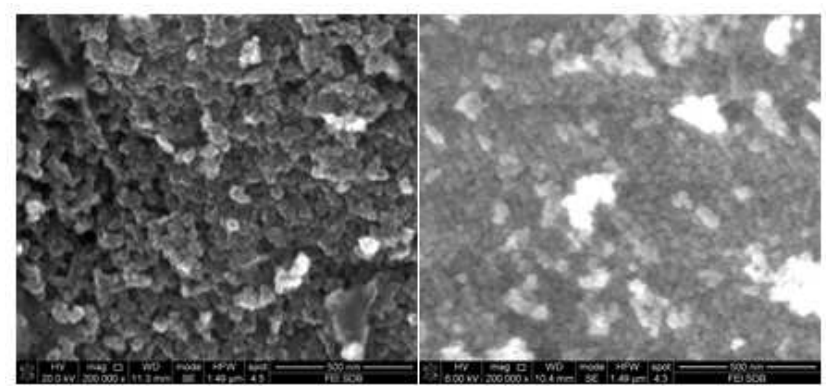

a. $2 \mathrm{~h}$

b. $2.5 \mathrm{~h}$

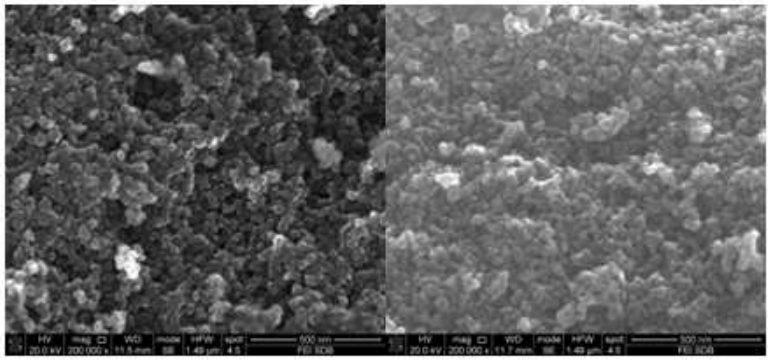

c. $3 \mathrm{~h}$

d. $3.5 \mathrm{~h}$

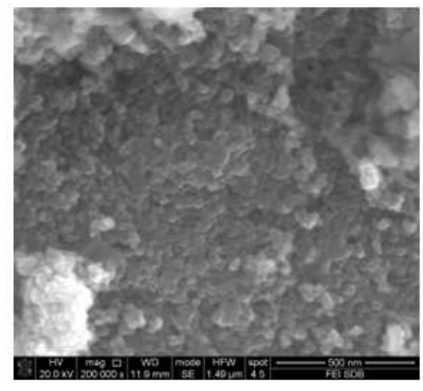

e. $4 \mathrm{~h}$

图5 不同煅烧时间催化剂的SEM图。

\section{2. 催化剂的 $\mathrm{NH}_{3}-\mathrm{SCR}$ 性能测试}

\subsection{1. 载体的活性测试}

图6为三种不同方式所制备载体的 $\mathrm{NH}_{3}-\mathrm{SCR}$ 活性测试 结果。从图中可以看出, 单独的 $\mathrm{TiO}_{2}-\mathrm{ZrO}_{2}$ 载体也具有一 定的活性, 且在 $80^{\circ} \mathrm{C} \sim 200^{\circ} \mathrm{C}$ 范围内, 随着温度的升高, 三 种方法制备的载体活性均呈上升趋势。其中 $\mathrm{TiO}_{2}-\mathrm{ZrO}_{2}(\mathrm{Y})$ 和 $\mathrm{TiO}_{2}-\mathrm{ZrO}_{2}(\mathrm{~F})$ 的活性相差不大, $80 \sim 200^{\circ} \mathrm{C}$ 的活性在 $20 \% \sim 25 \%$ 左右, $\mathrm{TiO}_{2}-\mathrm{ZrO}_{2}$ (D) 的催化效率明显提高, $80^{\circ} \mathrm{C}$ 时 $\mathrm{NO}_{\mathrm{x}}$ 转化率已达到 $30 \%, 200{ }^{\circ} \mathrm{C}$ 时接近 $45 \%$ 。

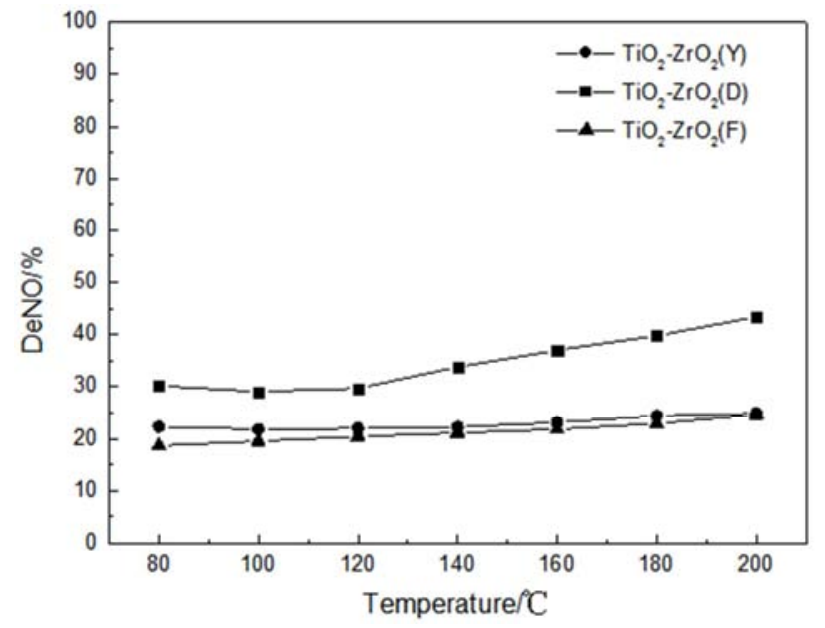

图6 不同载体的 $\mathrm{NH}_{3}-\mathrm{SCR}$ 性能测试图。

结合 XRD 与 SEM 表征可知, 这可能是因为 $\mathrm{TiO}_{2}-\mathrm{ZrO}_{2}(\mathrm{D})$ 载体粒径更小, 分散度更高, 可以提供更大 的比表面积, 促进反应气体与催化剂表面活性成分的接触 
和吸附。另外, $\mathrm{TiO}_{2}-\mathrm{ZrO}_{2}(\mathrm{D})$ 拥有更多的晶格缺陷和活性 位点。

\subsection{2. 不同㷽烧温度对催化剂性能的影响}

如图7, 图8所示为不同裧烧温度催化剂的活性测试和 抗硫性测试结果, 煅烧时间均为 $2.5 \mathrm{~h}$ 。从图7中可以看出, 在 $80^{\circ} \mathrm{C} \sim 100^{\circ} \mathrm{C}$ 之间, $200^{\circ} \mathrm{C}$ 煅烧的催化剂 $\mathrm{NO}$ 去除率明显高 于其它催化剂, $80^{\circ} \mathrm{C}$ 时为 $80.7 \%, 100^{\circ} \mathrm{C}$ 时达到 $98 \%$ 。 $120^{\circ} \mathrm{C}$ 之后, 除 $350^{\circ} \mathrm{C}$ 煅烧的催化剂以外, 其它催化剂的活性相 差不大，均达到 $95 \%$ 以上。图8所示为不同煅烧温度催化 剂在 $100^{\circ} \mathrm{C}$ 下的抗硫性测试结果。在持续通入 $8 \mathrm{~h}$ 的 $100 \mathrm{ppmSO}_{2}$ 之后, 陆烧温度为 $150^{\circ} \mathrm{C}, 250^{\circ} \mathrm{C}, 300^{\circ} \mathrm{C}$ 的催 化剂NOx转化率分别为 $33.9 \%, 36.5 \%, 66.4 \%$, 停止通入 $\mathrm{SO}_{2}$ 后继续下降至稳定, 说明 $\mathrm{SO}_{2}$ 对催化剂的毒化作用不可 逆。 $200^{\circ} \mathrm{C}$ 㷽烧的催化剂在抗硫 $8 \mathrm{~h}$ 后 $\mathrm{NO}_{\mathrm{x}}$ 基本维持在 $75 \%$ 左右, 其停止通入 $\mathrm{SO}_{2}$ 后恢复至 $82 \%$ 左右维持稳定, 说明 $\mathrm{SO}_{2}$ 对它的毒化作用部分可逆。

结合 $\mathrm{SEM}$ 表征结果, 煅烧温度为 $200^{\circ} \mathrm{C}$ 的催化剂粒径 较小且分散性更好, 更有利于SCR反应的进行。

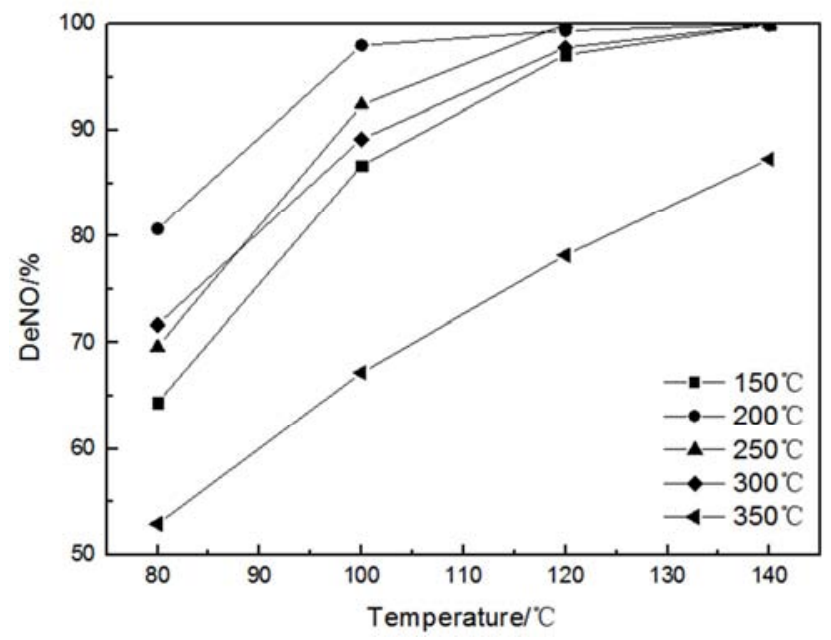

图7 不同搬烧温度催化剂的 $\mathrm{NH}_{3}$-SCR 性能测试。

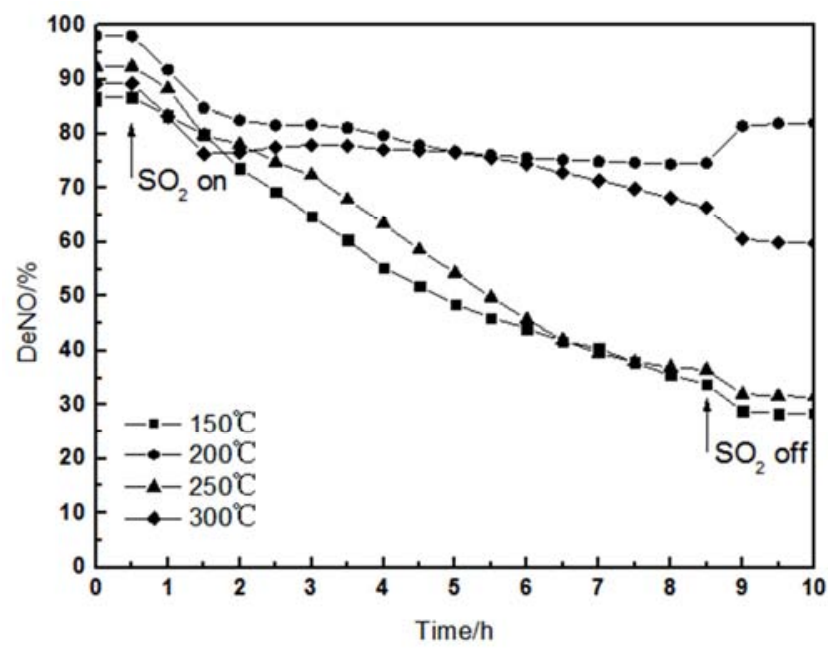

图8 不同殷烧温度催化剂的抗硫性测试。

\subsection{3. 不同煆烧时间对催化剂性能的影响}

如图9, 图10所示为不同㷽烧时间催化剂的活性测试 和抗硫性测试结果, 煅烧温度均为 $200^{\circ} \mathrm{C}$ 。从图中可以看 出, 炦烧时间从 $2 \mathrm{~h}$ 到 $4 \mathrm{~h}$, 催化剂在 $80^{\circ} \mathrm{C} \sim 140^{\circ} \mathrm{C}$ 内的 $\mathrm{NO}_{\mathrm{x}}$ 去除率整体上呈先增大后减小的趋势, $100^{\circ} \mathrm{C}$ 下的抗硫性 表现与活性测试一致。在 $100^{\circ} \mathrm{C}$ 时, 煅烧时间为 $2 \mathrm{~h}, 2.5 \mathrm{~h}$, $3 \mathrm{~h}, 3.5 \mathrm{~h}, 4 \mathrm{~h}$ 的催化剂活性分别为 $87.3 \%, 98 \%, 92.1 \%$, $95.8 \%$ 和 $85 \%$, 持续通入 $8 \mathrm{~h}$ 的 $100 \mathrm{ppmSO}_{2}$ 后 $\mathrm{NO}_{\mathrm{x}}$ 去除率分别 维持在 $44.6 \%, 75 \%, 49.1 \%, 52.4 \%, 39.5 \%$ 。炦烧时间为 $2.5 \mathrm{~h}$ 的催化剂表现最佳。

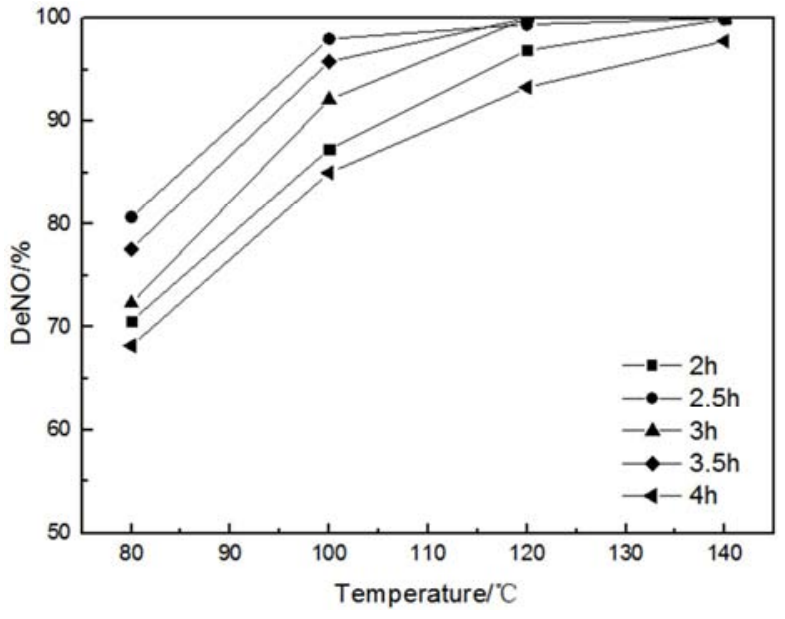

图9 不同煅烧时间催化剂的活性测试。

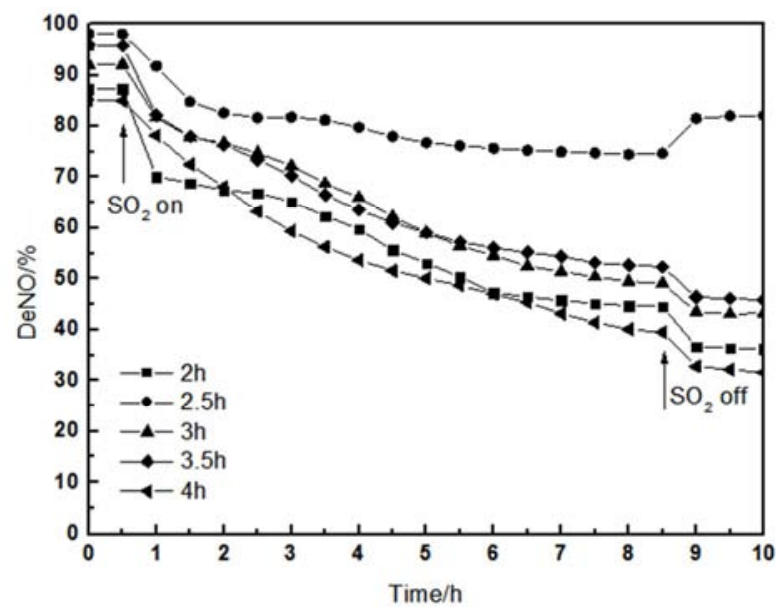

图10 不同㷽烧时间催化剂的抗硫性测试。

结合 SEM表征结果, 煅烧时间为 $2.5 \mathrm{~h}$ 的催化剂粒径较 小且分散性更好, 对SCR催化反应有利。

\section{4. 结论}

在本项研究中, 采用溶胶凝胶法制备载体, 分步浸渍 法制备催化剂。改进了 $\mathrm{TiO}_{2}-\mathrm{ZrO}_{2}$ 载体的制备方法, 有更 多的 $\mathrm{Zr}^{4+}$ 取代 $\mathrm{Ti}^{4+}{ }^{4}$ 参杂进入 $\mathrm{TiO}_{2}$ 晶格内, 产生了较多的晶格 缺陷和活性位点, 有利于催化活性的提高。通过改变催化 剂的煅烧温度和煅烧时间优化其SCR性能, 结果表明, 搬 烧温度为 $200^{\circ} \mathrm{C}$, 搬烧时间为 $2.5 \mathrm{~h}$ 的催化剂性能最佳, 
$100^{\circ} \mathrm{C}$ 的活性达到 $98 \%$, 持续通入 $100 \mathrm{ppm}$ 的 $\mathrm{SO}_{2} 8 \mathrm{~h}$ 后 $\mathrm{NO}_{\mathrm{x}}$ 去除率维持在 $75 \%$ 左右。

\section{致谢}

本文由“国家重点研发计划”资助, 项目编号: 2017YFC0210300。

\section{参考文献}

[1] Granger P, Parvulescu V I. Catalytic $\mathrm{NO}_{x}$ Abatement Systems for Mobile Sources: From Three-Way to Lean Burn after-Treatment Technologies [J]. Chemical Reviews, 2011, 111(5):3155.

[2] Dong W K, Nam K B, Hong S C. Influence of tungsten on the activity of a $\mathrm{Mn} / \mathrm{Ce} / \mathrm{W} / \mathrm{Ti}$ catalyst for the selective catalytic reduction of $\mathrm{NO}$ with $\mathrm{NH}_{3}$, at low temperatures [J]. Applied Catalysis A General, 2015, 497:160-166.

[3] Park E, Chin S, Kim J, et al. Preparation of $\mathrm{MnO}_{\mathrm{x}} / \mathrm{TiO}_{2}$ ultrafine nanocomposite with large surface area and its enhanced toluene oxidation at low temperature. Powder Technol, 2011, 208(3): 740.

[4] 卢熙宁, 宋存义, 童震松, 等. 锆掺杂 $\mathrm{TiO}_{2}$ 负载锰铈氧化物 低温催化还原 $\mathrm{NO}_{\mathrm{x}}[\mathrm{J}]$. 北京科技大学学报, 2014(9):1222-1232。
[5] Li J, Chang H, Ma L, et al. Low-temperature selective catalytic reduction of $\mathrm{NO}_{\mathrm{x}}$, with $\mathrm{NH}_{3}$, over metal oxide and zeolite catalysts-A review [J]. Catalysis Today, 2011, 175(1):147-156.

[6] Chen J P, Yang R T. Selective catalytic reduction of NO with $\mathrm{NH}_{3}$ on $\mathrm{SO}_{4}{ }^{2-} / \mathrm{TiO}_{2}$ superacid catalyst [J]. Journal of Catalysis, 1993, 139(1):277-288.

[7] 韩承辉, 刘炳华, 张惠良, 等. $\mathrm{TiO}_{2}-\mathrm{ZrO}_{2}$ 的表征及其异丙 醇催化转化性能[J]. 物理化学学报, 2006,22(8):993-998。

[8] Kazuhiro Ito, Shuichi Kakino, Keita Ikeue, et al. NO adsorption/desorption property of $\mathrm{TiO}_{2}-\mathrm{ZrO}_{2}$, having tolerance to $\mathrm{SO}_{2}$, poisoning [J]. Applied Catalysis B Environmental, 2007, 74(1-2):137-143.

[9] 王龙飞, 张亚平, 郭婉秋, 等. $\mathrm{WO}_{3} / \mathrm{TiO}_{2}-\mathrm{ZrO}_{2}$ 脱硝催化剂 制 备及其 $\mathrm{NH}_{3}$ 活化机理 [J]. 化工学报， 2015, 66(10):3903-3910.

[10] 张亚平, 汪小蕾, 孙克勤, 等. $\mathrm{WO}_{3}$ 对 $\mathrm{MnO}_{\mathrm{x}} / \mathrm{TiO}_{2}$ 低温脱硝 SCR 催化剂的改性研究 [J]. 燃料化学学报, 2011,39(10):782-786。

[11] 贠丽, 汪莉, 李强, 等. $\mathrm{Ce}-\mathrm{MnO}_{\mathrm{x}} / \mathrm{TiO}_{2}-\mathrm{ZrO}_{2}$ 低温选择催化 还原 NO 活性和抗毒性研究 [J]. 功能材料, 2016, 47(10):10059-10064。 